\title{
$\Omega$-stability for maps with nonwandering critical points
}

\author{
by
}

\author{
J. Delgado (Niterói), N. Romero (Barquisimeto), \\ A. Rovella (Montevideo) and F. Vilamajó (Terrasa)
}

\begin{abstract}
Sufficient conditions for a map having nonwandering critical points to be $\Omega$-stable are introduced. It is not known if these conditions are necessary, but they are easily verified for all known examples of $\Omega$-stable maps. Their necessity is shown in dimension two. Examples are given of Axiom A maps that have no cycles but are not $\Omega$-stable.
\end{abstract}

1. Introduction. Let $\operatorname{End}^{r}(M)$ denote the set of $C^{r}$ self mappings of a compact manifold $M$ endowed with the $C^{r}$ topology. Denote by $S(f)=$ $\left\{x: D f_{x}\right.$ is noninvertible $\}$ the set of critical points of a mapping $f$. Hyperbolic sets are defined here as for diffeomorphisms but with an additional condition: the restriction of $f$ to the set is either expanding or one-to-one. Other definitions are often used by different authors, but this one is better adapted to the study of stability; see the next section for a more complete discussion. An endomorphism $f$ is Axiom A if the set $\Omega(f)$ of nonwandering points is hyperbolic and the set of periodic points is dense in $\Omega(f)$. For an Axiom A map there exists a spectral decomposition of $\Omega(f)$ into basic sets, so the no cycles condition makes sense also for endomorphisms satisfying Axiom A. The problem of stability of endomorphisms is still far from being well understood.

For maps $f$ with $S(f) \cap \Omega(f)=\emptyset$ the following result holds: $f$ is Axiom $A$ with no cycles if and only if it is $\Omega$-stable. The sufficiency of the conditions was proved by Przytycki [P1] in 1976, while the converse is due to Aoki, Moriyasu and Sumi [AMS] in 2001.

Allowing the presence of nonwandering critical points, Ikeda proved in [I] that if $f$ is Axiom A and satisfies the no cycles condition, then $f$ is $\Omega$-inverse limit stable, a weaker form of stability. Mañé and Pugh [MP] gave an example of an $\Omega$-stable map satisfying $\Omega(f) \cap S(f) \neq \emptyset$ persistently. However, as

2000 Mathematics Subject Classification: Primary 37D20.

Key words and phrases: $\Omega$-stable, hyperbolic set, Axiom A, no cycles condition. 
will be shown in Section 3, one cannot expect $\Omega$-stability for every Axiom A map without cycles. In this article a condition will be presented which, when imposed on those basic sets of $\Omega(f)$ that contain critical points, implies the $\Omega$-stability of $f$.

Definition 1. Let $\Lambda$ be a compact invariant set for $f \in \operatorname{End}^{r}(M)$, $r \geq 1$. Say that $f$ satisfies condition $C$ in $\Lambda$ if there exists a continuous invariant splitting $T_{\Lambda} M=E^{\mathrm{ss}} \oplus E^{\mathrm{c}}$ such that the following holds:

(1) There exist constants $K>0$ and $\lambda \in(0,1)$ such that

$$
\left\|D f_{x}^{n}(v)\right\| \leq K \lambda^{n} \quad \text { and } \quad \frac{\left\|D f_{x}^{n}(v)\right\|}{\left\|D f_{x}^{n}(w)\right\|} \leq K \lambda^{n},
$$

for every $n>0, x \in \Lambda$ and unit vectors $v \in E^{\text {ss }}$ and $w \in E^{\mathrm{c}}$ with $\left\|D f_{x}^{n}(w)\right\| \neq 0$.

(2) There exists $\varepsilon>0$ such that $\Lambda \cap W_{\varepsilon}^{\text {ss }}(x)=\{x\}$ for every $x \in \Lambda$.

Some comments are in order here. If the set $\Lambda$ is hyperbolic for $f$, it is known that a continuous splitting $T_{\Lambda} M=E^{\mathrm{s}} \oplus E^{\mathrm{u}}$ already exists. The first condition obviously implies that $\operatorname{Ker} D f_{x} \subset E_{x}^{\text {ss }} \subset E_{x}^{\text {s }}$ for every $x \in \Lambda$. In this case, the central direction may contain part of $E^{\mathrm{s}}$, but $E^{\mathrm{ss}}$ cannot contain part of $E^{\mathrm{u}}$ by the first assertion of condition (1). Whenever the first condition of the definition holds, the strong stable manifolds exist; these were denoted $W_{\varepsilon}^{\text {ss }}(x)$ in the second condition. If the set $\Lambda$ does not contain critical points, then condition $\mathrm{C}$ is irrelevant to our purposes.

The main result of this paper is the following theorem, which will be proved in Section 3:

TheOREm 1. If $f$ is Axiom A, satisfies the no cycle condition and each basic set containing critical points satisfies condition $C$, then $f$ is $C^{1} \Omega$ stable.

These hypotheses are satisfied by the $\Omega$-stable maps given by Mañé and Pugh in [MP]. It is still not known if these conditions are also necessary for $\Omega$-stability when the dimension of $M$ is greater than two. In Section 2 we give some preliminary definitions and three examples: the first one is taken from [P2], it serves to explain the definition of a hyperbolic set for an endomorphism, and illustrates the theorems on $\Omega$-inverse stability. The second one is a sketch of the example given in $[\mathrm{MP}]$ and mentioned above. The third one gives an Axiom A map without cycles that is not $\Omega$-stable due to the presence of critical points, answering negatively the following question posed in [I]: does Axiom A and no cycles imply $\Omega$-stability?

In Section 3 the proof of the theorem is given and in Section 4 it is shown that if $M$ has dimension 2, then the $C^{1} \Omega$-stability of an Axiom A map $f$ implies that $\Lambda \cap S(f)=\emptyset$ if $\Lambda$ is a basic piece of $\Omega(f)$ with infinitely many 
points. The necessity of condition $\mathrm{C}$ for $C^{1} \Omega$-stability follows trivially in the case that $M$ is two-dimensional.

We want to thank Martín Sambarino for useful suggestions and comments.

2. Preliminaries. The following is the definition of a hyperbolic set for an endomorphism $f \in \operatorname{End}^{r}(M)$; it was introduced by Mañé in [M].

Definition 2. Let $\Lambda$ be a compact set such that $f(\Lambda)=\Lambda$. Then $\Lambda$ is called hyperbolic for $f$ if there exists a continuous splitting $T_{\Lambda} M=E^{\mathrm{s}} \oplus E^{\mathrm{u}}$ such that:

(1) $D f\left(E^{\mathrm{s}}\right) \subset E^{\mathrm{s}}$ and $D f\left(E^{\mathrm{u}}\right)=E^{\mathrm{u}}$.

(2) $D f$ uniformly contracts vectors in $E^{\mathrm{s}}$ and uniformly expands vectors in $E^{\mathrm{u}}$.

(3) If $x, y \in \Lambda$ and $f(x)=f(y)=z$, then $E_{z}^{\mathrm{s}}=\{0\}$.

The first difference with the definition of a hyperbolic set for a diffeomorphism is that $D f_{x}\left(E_{x}^{\mathrm{s}}\right)$ may be strictly contained in $E_{f(x)}^{\mathrm{s}}$ (this will certainly occur when $x$ is a critical point). The main difference, however, is the third condition. For example, for transitive $\Lambda$, it implies that either the map is injective in $\Lambda$ or $E_{x}^{\mathrm{s}}=\{0\}$ for every $x \in \Lambda$. A hyperbolic set satisfying this last condition is called quasi-expanding. The first example below will show that a map satisfying items (1) and (2) of the above definition but not (3) is not $\Omega$-stable. The main reason why condition (3) was included becomes clear from the work of Przytycki in [P1] and [P2]. The definition he gave of a hyperbolic set does not contain condition (3), and then the unstable direction of a point $x$ could depend on the preorbits of the point. The definition of a hyperbolic set in $[\mathrm{P} 1]$ is the following (as in $[\mathrm{I}]$, it will be called P-hyperbolic here):

Definition 3. A set $\Lambda$ is P-hyperbolic for $f$ if it is compact, invariant, and for every $f$-orbit $\left\{x_{n}: n \in \mathbb{Z}\right\} \subset \Lambda$ there exists an invariant decomposition of the tangent space at the point $x_{0}$ :

$$
T_{x_{0}} M=E_{\left\{x_{n}\right\}}^{\mathrm{s}} \oplus E_{\left\{x_{n}\right\}}^{\mathrm{u}}
$$

where $E_{\left\{x_{n}\right\}}^{\mathrm{s}}$ is uniformly contracted, and $E_{\left\{x_{n}\right\}}^{\mathrm{u}}$ uniformly expanded.

Here the invariance of the decomposition means that

$$
D f_{x_{0}}\left(E_{\left\{x_{n}\right\}}^{s, u}\right) \subset E_{\left\{f\left(x_{n}\right)\right\}}^{s, u} \subset T_{f\left(x_{0}\right)} M .
$$

It is easily verified that if $\Lambda$ is a transitive $\mathrm{P}$-hyperbolic set that satisfies the third condition in the definition of a hyperbolic set, then $\Lambda$ is hyperbolic.

Przytycki also showed that this kind of hyperbolicity implies inverse stability (under the assumption $S(f) \cap \Omega(f)=\emptyset$ ) and also the existence 
of invariant manifolds (the unstable manifold of a point $x$ depends on the preorbit).

Definition 4. A map $f \in \operatorname{End}^{r}(M)$ satisfies Axiom $A$ if $\Omega(f)$ is hyperbolic and the set of periodic points of $f$ is dense in $\Omega(f)$.

Basic sets and cycles can be defined as for the case of diffeomorphisms. A hyperbolic set $\Lambda$ is called a basic set of $f$ provided it is transitive and locally maximal (that is, $\bigcap_{n \in \mathbb{Z}} f^{n}(U)=\Lambda$ for some neighborhood $U$ of $\Lambda$ ). The homoclinic classes are defined exactly as for the case of diffeomorphisms. For Axiom A maps the nonwandering set is a finite union of basic sets, each of which is the union of a homoclinic class and its future iterates (which are finite in number because the set of homoclinic classes is finite). Also, as in the case of diffeomorphisms, if a hyperbolic set (or P-hyperbolic set) $\Lambda$ has a dense subset of periodic points, then it has a local product structure; see Section 2 in [I] and Proposition 3.9 in [P1].

For an invariant set $\Lambda$ of a map $f$, the inverse limit set $\widetilde{\Lambda}_{f}$ of $\Lambda$ with respect to $f$ is defined as the set of sequences $\left\{x_{n}\right\}_{n \in \mathbb{Z}} \subset \Lambda$ such that $f\left(x_{n}\right)=x_{n+1}$ for every $n$. If $\widetilde{\Lambda}_{f}$ is given the product topology, the shift map $\sigma_{f}$ is a homeomorphism of $\widetilde{\Lambda}_{f}$, and the map $\left\{x_{n}\right\} \mapsto x_{0}$ is a semiconjugacy between $\sigma$ and $f$. Finally, $f$ is called $C^{r} \Omega$-inverse stable if there exists a neighborhood $U$ of $f$ such that $\sigma_{g}$ restricted to the inverse limit set of $\Omega(g)$ is conjugate to $\sigma_{f}$ restricted to the inverse limit set of $\Omega(f)$.

We now state some known theorems about sufficient conditions for $\Omega$ stability. The first one is taken from the work of Przytycki and the second is due to Ikeda.

Theorem 2 (Przytycki [P1], [P2]). If $\Omega(f)$ is P-hyperbolic, the periodic points of $f$ are dense in $\Omega(f), f$ has no critical points and $\Omega(f)$ has no cycles, then:

(a) $f$ is $\Omega$-inverse stable.

(b) $f$ is $\Omega$-stable if and only if $\Omega(f)$ is hyperbolic.

Theorem 3 (Ikeda [I]). If $f$ is Axiom $A$ and there are no cycles, then $f$ is $\Omega$-inverse stable.

The theorem stated in the introduction is partially based on the proofs of these results and generalizes both.

\subsection{Examples}

EXAmple 1. Let $f$ be the map defined in $S^{1} \times \mathbb{R}$ by $f(z, y)=\left(z^{2}, a y\right)$, where $a \in \mathbb{R}$ has absolute value less than 1 . Then the set $\Lambda=S^{1} \times\{0\}$ is compact, invariant, and satisfies the first and second conditions in the definition of a hyperbolic set, but does not satisfy the third one $(\Lambda$ is $\mathrm{P}$ hyperbolic but not hyperbolic). The reason why this set is not included in the 
definition of hyperbolic is that it is not stable under perturbations: indeed, there exist maps arbitrarily close to $f$ for which there is no continuous splitting satisfying (1) and (2) in the definition above. In fact, if $\varepsilon$ is small, then for every $g$ in a neighborhood of $f$ we have $g(A) \subset A$, where $A=$ $S^{1} \times(-\varepsilon, \varepsilon)$. There exists $g$ close to $f$ and $n>0$ such that for some $z_{0}$, the intersection $\left\{(z, y): z=z_{0}\right\} \cap g^{n}(A)$ is not connected. For example, let $g(z, y)=\left(z^{2}, h(z, y)\right)$, and suppose that $h$ satisfies the following conditions:

1. $|h(z, y)| \leq \varepsilon_{0}<\varepsilon$ for every $|y|<\varepsilon$.

2. $h(-1, y)<0$ for every $|y|<\varepsilon$.

3. $h(1, y)>0$ for every $|y|<\varepsilon$.

If we let $H_{0}=\left\{\left(e^{i \theta}, y\right) \in A: 0 \leq \theta \leq \pi\right\}$ and $H_{1}=\left\{\left(e^{i \theta}, y\right) \in A\right.$ : $\pi \leq \theta \leq 2 \pi\}$, then $g\left(H_{0}\right)$ and $g\left(H_{1}\right)$ are both homeomorphic to an annulus and have intersection with nonempty interior; however, $g\left(H_{0}\right) \cap g\left(H_{1}\right)$ $\cap\{z=1\}=\emptyset$. This implies that the set $\Lambda(g)=\bigcap_{k>0} g^{k}(A)$ is not a simple closed curve; it is connected and its inverse limit is still a solenoid, as is the case for $f$, according to Theorem 2 above. But the restriction of $g$ to $\Lambda(g)$ is not injective. Therefore it is not possible to define a uniquely determined unstable direction at each point; actually, there exists one unstable direction for each distinct preorbit of a point. See the final part of the article of Przytycki [P2] where this and other examples are studied. This kind of examples appeared in recent articles, where the solenoidal attractors are considered from the ergodic $[\mathrm{T}]$ and topological $[\mathrm{BKR}]$ viewpoints.

EXAMPLE 2. Let $M$ be the product of a torus $T$ and a circle $S^{1}$ and define $f$ in $M$ by $f(x, y)=(A x, h(x, y))$, where $A$ is an Anosov diffeomorphism and $h_{x}(y)=h(x, y)$ satisfies the following conditions:

(1) For every $x \in T, h_{x}$ has an attracting fixed point at 1 ; there exist $x_{0}, x_{1} \in T$ such that $h_{x_{0}}^{\prime}(1)>0$ and $h_{x_{1}}^{\prime}(1)<0$.

(2) For every $x \in T$, the point -1 is a repelling fixed point of $h_{x}$.

(3) The derivative of $h_{x}$ at -1 is greater than any possible expansion of $A$ in $T$, and the derivative of $h_{x}$ at 1 is less than the contraction of $A$ in $T$.

(4) For any positive $A$-orbit $x_{n}$ in $T$ and any $y \neq-1$ in $S^{1}$ the sequence $z_{n}$ defined by $z_{0}=y$ and $z_{n+1}=h_{x_{n}}\left(z_{n}\right)$ converges to 1 .

It is clear that such a function $h$ can be constructed of class $C^{\infty}$. Then the following facts are true:

- Conditions (3) and (4) above imply that $\Omega(f)=\omega_{-1} \cup \omega_{1}$, where $\omega_{i}=T \times\{i\}$ are the basic sets of $\Omega(f)$.

- $\omega_{1}$ contains critical points of $f$, because property (1) of $h$ implies that there exists $z \in T$ such that $h_{z}^{\prime}(1)=0$. 
- Condition $\mathrm{C}$ is satisfied because $\omega_{-1}$ does not contain critical points of $f$. For every $(x, y) \in \omega_{1}$ the vector $(0,1)$, transverse to the submanifold $\omega_{1}$, generates a subspace $E_{(x, y)}^{\mathrm{ss}}$ as in Definition 1 .

Therefore Theorem 1 implies that $f$ is $\Omega$-stable. A direct proof is also very simple (see $[\mathrm{MP}]$ ). Moreover, if $g$ is a small perturbation of $f$, then there exists a submanifold $N$ close to $\omega_{1}$ that is a basic set of $g$ and contains critical points: indeed, the vector $(0,1)$ is transverse to $N$, and the coordinate of $D g_{z}(0,1)$ in the direction of $(0,1)$ is sometimes positive and sometimes negative. This is the example given in [MP]. It follows that $S(f) \cap \Omega(f) \neq \emptyset$ persistently.

EXAMPLE 3. In this example we construct a horseshoe with singularities. A usual horseshoe can be defined in the square $Q=[0,1]^{2}$, having the form $(x / 3,3 y)$ if $y \leq 1 / 3,(1-x / 3,3(1-y))$ if $2 / 3 \leq y \leq 1$ and such that the image of $1 / 3 \leq y \leq 2 / 3$ is disjoint from $Q$.

Define $f$ as above but instead of $x / 3$ put a function $h(x)=x^{2} / 3$ for $y \leq$ $1 / 3$ and $1-x^{2} / 3$ for $2 / 3 \leq y \leq 1$. Then the map is extended to the sphere, in such a way that the set of critical points is a line containing the segment $\{(x, y): x=0,0 \leq y \leq 1\}$. As for the case of the usual horseshoe, the nonwandering set is a Cantor set $\Lambda$ (the horseshoe) contained in $Q$, plus two fixed points, one of them attracting and the other one repelling (observe that the points in $\Omega$ have preimages outside $Q$, but these are wandering points). Now perturb $f$ in such a way that the new map $g$ is equal to $f$ outside a neighborhood of the origin and has the form $g_{a}(x, y)=\left(h_{a}(x), 3 y\right)$ where $h_{a}(x)=x^{2} / 3-a x$ with $a>0$ in a small neighborhood of 0 . The origin 0 is still a fixed point of $g_{a}$ and it is clear that the local invariant manifolds at 0 remain unchanged. Therefore the point $(1,0)$ is still homoclinic for every perturbation: it belongs to the stable and unstable manifolds of the origin. Let $x_{n}(a)$ be such that $g_{a}^{n}(1,0)=\left(x_{n}(a), 0\right)$ for every $n>0$, where $x_{n}(a)$ converges to 0 (uniformly in $a$ ) as $n \rightarrow \infty$. Finally, given $a$ small, choose $n$ so that $x_{n}(a)<3 a$ and then fix $n$ and diminish $a$ to obtain $x_{n}(a)=3 a$. It follows that $(3 a, 0) \in \Omega\left(g_{a}\right)$, and $g_{a}(3 a, 0)=(0,0)$, so the restriction of $g_{a}$ to this basic piece is not injective, therefore $g_{a}$ and $f$ cannot be $\Omega$-conjugate. The reason why this map $f$ works as an example of an Axiom A map without cycles that is not $\Omega$-stable is that the kernel of the differential of $f$ at the critical point at the origin cannot be made disjoint from the directions $X-Y$ for $X$ and $Y$ in $\Omega(f)$. This is exactly what condition $\mathrm{C}$ persistently avoids.

3. Proof of the theorem. Begin with a mapping $f$ that satisfies the hypothesis of the theorem. As $f$ is Axiom A, there exists a spectral decomposition of $\Omega(f)$ into a finite number of basic sets, say $\Omega(f)=\bigcup \Lambda_{j}$. An important fact that will be used in the proof is that there exists a $C^{1}$ neigh- 
borhood $\mathcal{U}$ of $f$ such that every $\Lambda_{j}$ has a continuation in $\mathcal{U}$. This is proved as for the case of diffeomorphisms. In addition, the no cycles condition implies that the union of these sets constitutes the whole nonwandering set of the perturbation. Still more important is the fact that every continuation is P-hyperbolic. This was proved by Ikeda (cf. [I, Theorem 2.2]). The precise formulation is the following:

Proposition 1. Given an Axiom A map $f \in \operatorname{End}^{r}(M)$ with spectral decomposition $\Omega(f)=\bigcup \Lambda_{j}$, there exist a neighborhood $\mathcal{U}$ of $f$ in $\operatorname{End}^{r}(M)$ and, for every $j$, a continuous mapping $\varphi_{j}: \mathcal{U} \rightarrow C^{0}\left(\Lambda_{j}, M\right)$ such that:

(1) $\varphi_{j}(f)$ is the inclusion.

(2) If $\Lambda_{j}(g)=\varphi_{j}(g)\left(\Lambda_{j}\right)$, then $g \varphi_{j}(g)=\varphi_{j}(g) f$ on $\Lambda_{j}$, so $\varphi_{j}(g)$ is a semiconjugacy from $\Lambda_{j}$ onto $\Lambda_{j}(g)$.

(3) The $C^{0}$ distance from $\varphi_{j}(g)$ to the inclusion goes to zero as $g$ converges to $f$ in the $C^{0}$ topology.

(4) The nonwandering set of $g$ is the union of the sets $\Lambda_{j}(g)$.

(5) For each $j$, the set $\Lambda_{j}(g)$ is P-hyperbolic for $g$.

The main difference with the case of diffeomorphisms is that in the latter case $\varphi_{j}(g)$ is one-to-one and $\Lambda_{j}(g)$ is hyperbolic for $g$. This is not the case for endomorphisms as Example 3 of the previous section shows. For diffeomorphisms the $\varphi_{j}$ establish the conjugacy realizing the $\Omega$-stability of $f$. Ikeda used these semiconjugacies to obtain true conjugacies between the maps induced by $f$ and $g$ in the respective inverse limit sets of $\Lambda_{j}$ and $\Lambda_{j}(g)$.

As already explained, if $f$ is Axiom A, either every basic set $\Lambda$ is quasi expanding or the restriction of $f$ to $\Lambda$ is injective. In the first case it is clear that $\Lambda$ does not contain critical points and that the restriction of $f$ to $\Lambda$ is conjugate to the restriction of $g$ to $\Lambda(g)$. So assume from now on that the restriction of $f$ to $\Lambda$ is injective and that $f$ satisfies condition $\mathrm{C}$ on $\Lambda$. The next, fundamental step in the proof of the theorem is the following:

Proposition 2. If $\Lambda$ is a basic set for $f$ and satisfies condition $C$, then there exists a $C^{1}$ neighborhood of $f$ such that every $g$ in that neighborhood is injective in $\Lambda(g)$.

Using these two propositions it is easy to prove Theorem 1:

Proof of Theorem 1. If $g$ is a small $C^{1}$ perturbation of $f$ then by Proposition $1, \Omega(g)=\bigcup \Lambda_{j}(g)$. If $\Lambda_{j}=\Lambda_{j}(f)$ is expanding, then $f \mid \Lambda_{j}$ is conjugate to $g \mid \Lambda_{j}(g)$. If $\Lambda_{j}$ is not expanding then by Proposition $2, g$ is injective in $\Lambda_{j}(g)$. So the inverse limit of $g$ in $\widetilde{\Lambda}_{j}(g)$ is trivially conjugate to $g \mid \Lambda_{j}(g)$. From a theorem of Ikeda (Theorem 3 in Section 2) it follows that the inverse limits of $f$ and $g$ in $\Lambda_{j}(f)$ and $\Lambda_{j}(g)$ are conjugate. Thus $f \mid \Lambda_{j}(f)$ is conjugate to $g \mid \Lambda_{j}(g)$. 
It remains to prove Proposition 2. The following sequence of results is needed to prove the local injectivity of $g$ on $\Lambda(g)$, which will be finally achieved in Lemma 4 below; using this, it is easily shown that $g$ is injective in $\Lambda(g)$.

The first result is a generalization of the inverse function theorem in the neighborhood of a critical point $x$. The question is: for which pairs of points $y$ and $z$ close to $x$ is it true that $F(y)=F(z)$ ?

Definition 5. A subset $\mathcal{C}$ of $\mathbb{R}^{n}$ is a cone if $t v \in \mathcal{C}$ for every $v \in \mathcal{C}$ and $t \in \mathbb{R}$. The interior of a cone is defined as the union of its topological interior and the origin, so that the interior of a cone is also a cone. Denote by $\operatorname{int}(\mathcal{C})$ the interior of the cone $\mathcal{C}$.

Lemma 1. Let $F: \mathbb{R}^{n} \rightarrow \mathbb{R}^{n}$ be a $C^{1}$ map having a critical point at 0 . Given a closed cone $\mathcal{C}$ containing the kernel of DF at 0 in its interior, there exists some $\delta_{0}>0$ such that:

(a) For points $y, z$ in the ball of center 0 and radius $\delta_{0}$, the condition $F(y)=F(z)$ implies that $y-z \in \mathcal{C}$.

(b) If , in addition, the closure of $D_{0}^{-1}(\mathcal{C})$ is contained in $\operatorname{int}(\mathcal{C})$ and $F(0)=0$, then $\delta_{0}$ can be chosen to satisfy also

$$
F^{-1}(\mathcal{C}) \cap B\left(0 ; \delta_{0}\right) \subset \operatorname{int}(\mathcal{C}) .
$$

Proof. Observe first that given $\mathcal{C}$ there exists $\alpha>0$ such that

$$
\left\|D F_{0}(v)\right\| \geq \alpha\|v\| \quad \text { whenever } v \notin \mathcal{C} .
$$

Next, since $F$ is of class $C^{1}$ there exists a number $\delta_{0}>0$ such that, for every $y, z \in B\left(0 ; \delta_{0}\right)$,

$$
\left\|F(y)-F(z)-D F_{z}(y-z)\right\| \leq \frac{\alpha}{4}\|y-z\|,
$$

and for any vector $v$,

$$
\left\|D F_{y}(v)-D F_{z}(v)\right\| \leq \frac{\alpha}{4}\|v\| .
$$

Now let $y, z \in B\left(0 ; \delta_{0}\right)$; from (2) and (3) it follows that

$$
\begin{aligned}
\left\|F(y)-F(z)-D F_{0}(y-z)\right\| \leq & \left\|F(y)-F(z)-D F_{z}(y-z)\right\| \\
& +\left\|D F_{z}(y-z)-D F_{0}(y-z)\right\| \\
\leq & \frac{\alpha}{2}\|y-z\| .
\end{aligned}
$$

This together with (1) implies that if $y-z \notin \mathcal{C}$, then $\|F(y)-F(z)\| \geq\left\|D F_{0}(y-z)\right\|-\left\|F(y)-F(z)-D F_{0}(y-z)\right\| \geq \frac{\alpha}{2}\|y-z\|$, which clearly implies $F(y) \neq F(z)$.

To prove part (b) observe that if a $C^{1}$ curve $\alpha$ in $\mathbb{R}^{n}$ such that $\alpha(0)=0$ satisfies $\alpha^{\prime}(t) \notin \mathcal{C}$ for every $t>0$, then $\alpha(t) \notin \mathcal{C}$ for every $t>0$. Suppose that $V \notin \operatorname{int}(\mathcal{C})$ has small norm $\|V\|<\delta_{0}$. Observe that by hypothesis 
$D F_{0}(V) \notin \mathcal{C}$; moreover, the distance from $D F_{0}(V) /\left\|D F_{0}(V)\right\|$ to the cone is greater than a positive constant $\varrho_{0}$ independent of $V$. The lemma will be proved if we show that $F(V) \notin \operatorname{int}(\mathcal{C})$.

Consider the curve $\alpha(t)=F(t V)$. Observe that given $\varrho>0$ there exists $\delta_{0}$ such that

$$
\left\|D F_{z}(W)-D F_{0}(W)\right\| \leq \varrho\|W\|
$$

for $z \in B\left(0 ; \delta_{0}\right)$ and any vector $W$. For every vector $w \neq 0$ we denote by $n(w)$ the unit vector $w /\|w\|$. An easy calculation using (1), (3) and (4) shows that

$$
\left\|n\left(D F_{0}(V)\right)-n\left(D F_{t V}(V)\right)\right\| \leq \frac{8 \varrho}{3 \alpha^{2}} .
$$

So take $\varrho<3 \alpha^{2} \varrho_{0} / 8$ and the corresponding $\delta_{0}$ to obtain $\alpha^{\prime}(t)=D F_{t V}(V) \notin$ $\mathcal{C}$ for every $t>0$, which implies that $F(V)=\alpha(1) \notin \mathcal{C}$, as claimed.

The next step in the proof of Proposition 2 is to show that every $C^{1}$ small perturbation $g$ of $f$ also satisfies condition $\mathrm{C}$ when restricted to $\Lambda(g)$. Note that the existence of strong stable directions is an open $C^{1}$ condition; this implies that $g \mid \Lambda(g)$ satisfies the domination condition.

The construction of invariant manifolds can be done by small changes of the techniques used for the case of diffeomorphisms (see for example [P1, Section 2] or [I, Section 2.3]). However, just for further reference, we will now describe one of the possible constructions of the strong stable manifolds. Consider a P-hyperbolic set $\Lambda(g)$ of $g$ such that the first condition of Definition 1 is satisfied on $\Lambda$ (that is, there exists a dominating strong stable direction). The local strong stable set of a point $x \in \Lambda(g)$ is defined as the set of points $y$ in an $\varepsilon$-neighborhood of $x$ such that the distance between the iterates of $x$ and $y$ decreases exponentially with a rate very close to the $\lambda$ of the strong contraction condition in Definition 1.

Denote by $\exp _{x}$ the exponential map from $T_{x} M$ to $M$. There exists some $\varepsilon_{0}>0$ such that, for every $x \in M$, the map $\exp _{x}$ is a diffeomorphism from the ball $B\left(0 ; \varepsilon_{0}\right)$ in $T_{x} M$ to $B\left(x ; \varepsilon_{0}\right)$ in $M$.

Let $\left\{\mathcal{C}_{x} \subset T_{x} M: x \in \Lambda\right\}$ be a continuous field of closed cones such that each $\mathcal{C}_{x}$ contains $E_{x}^{\text {ss }}$ in its interior and $\mathcal{C}_{x} \cap E_{x}^{\mathrm{c}}=\{0\}$. Define

$$
\widetilde{\mathcal{C}}_{x}=\exp _{x}\left(\mathcal{C}_{x}\right) \quad \text { and } \quad \operatorname{int}\left(\widetilde{\mathcal{C}}_{x}\right)=\exp _{x}\left(\operatorname{int}\left(\mathcal{C}_{x}\right)\right) .
$$

For $x$ in $\Lambda$ define

$$
\widetilde{\mathcal{C}}_{x}^{0}\left(\varepsilon_{0}\right)=\exp _{x}\left(\mathcal{C}_{x} \cap B\left(0 ; \varepsilon_{0}\right)\right) \subset B\left(x ; \varepsilon_{0}\right)
$$

and for $m>0$, by induction,

$$
\widetilde{\mathcal{C}}_{x}^{m}\left(\varepsilon_{0}\right)=g^{-1}\left(\widetilde{\mathcal{C}}_{g(x)}^{m-1}\left(\varepsilon_{0}\right)\right) \cap B\left(x ; \varepsilon_{0}\right) .
$$

It is clear that the domination condition (together with the fact that $\Lambda(g)$ is compact) implies that there exists an $m_{0}$ such that $D g_{x}^{-m}\left(\mathcal{C}_{g^{m}(x)}\right)$ is con- 
tained in the interior of $\mathcal{C}_{x}$ for every $m \geq m_{0}$ and $x \in \Lambda(g)$ (and converges to $E_{x}^{\text {ss }}$ as $\left.m \rightarrow \infty\right)$. Therefore the same property holds in the ambient manifold; indeed, part (b) of Lemma 1 implies that for $m=m_{0}$,

$$
\widetilde{\mathcal{C}}_{x}^{m}\left(\varepsilon_{0}\right) \subset \operatorname{int}\left(\widetilde{\mathcal{C}}_{x}\right) .
$$

Then (7) holds for every $m=k m_{0}, k>0$, and also for every $m \geq k_{0} m_{0}$ for some positive $k_{0}$, by equicontinuity of $D g_{x}^{j}, j \in\left\{1, \ldots, m_{0}-1\right\}, x \in \Lambda$. The intersection of these sets gives the local strong stable set of $x$ :

$$
\bigcap_{m \geq 0} \widetilde{\mathcal{C}}_{x}^{m}\left(\varepsilon_{0}\right)=W_{\varepsilon_{0}}^{\mathrm{ss}}(x) \cap B\left(x ; \varepsilon_{0}\right),
$$

and it can be shown that the local strong stable set is a manifold tangent to $E_{x}^{\mathrm{ss}}$ at $x$.

Note also that by the construction of an adapted Riemannian metric, one can suppose without loss of generality that $m_{0}=1$.

Remark 1. For $\varepsilon_{0}$ defined above, note that by item (3) of Proposition 1 , there exists some positive constant $\delta$ such that $d(x, y) \geq \varepsilon_{0}$ implies that $d(g(x), g(y)) \geq \delta$, whenever $x$ and $y$ are in $\Lambda(g)$. This is clear since the same holds for $f$. When $\Lambda(g)$ is a P-hyperbolic set of $g$, the restriction of $g$ to $\Lambda(g)$ is expansive, in the sense that there exists some positive constant $\delta_{0}$ such that for any two whole orbits $\left\{x_{n}\right\}$ and $\left\{y_{n}\right\}$ of $g$ in $\Lambda(g)$, there exists some $n$ such that $d\left(x_{n}, y_{n}\right)>\delta_{0}$. Obviously one can take $\delta=\delta_{0}$. See [P1] and $[\mathrm{I}]$. Such a $\delta$ is called a constant of expansivity.

Now observe that for any two whole orbits as above such that $y_{0} \in$ $W_{\varepsilon_{0}}^{\mathrm{s}}\left(x_{0} ; g\right)$, there exists a nonpositive integer $n$ such that $d\left(x_{n}, y_{n}\right) \in\left(\delta, \varepsilon_{0}\right)$. Indeed, if $d\left(x_{0}, y_{0}\right)<\delta$, then the same holds for future iterates, so there exists a first negative index $n$ such that $d\left(x_{n}, y_{n}\right)>\delta$. Hence $d\left(x_{n+1}, y_{n+1}\right) \leq$ $\delta$, which implies that $d\left(x_{n}, y_{n}\right)<\varepsilon_{0}$, as claimed.

Lemma 2. Suppose that $f$ satisfies condition $C$ in $\Lambda$. Then there exists a $C^{1}$ neighborhood $\mathcal{U}$ of $f$ such that for every $g \in \mathcal{U}$, the continuation $\Lambda(g)$ of $\Lambda$ has a well defined strong contracting direction $E_{x}^{\text {ss }}(g)$. The local strong stable manifold of any point $x \in \Lambda(g)$ intersects $\Lambda(g)$ only at $\{x\}$. In other words, $g$ satisfies condition $C$ in $\Lambda(g)$.

Proof. It remains to prove the last assertion of the lemma; suppose by contradiction that there exist a number $\delta>0$ sequences $\left\{x_{n}\right\},\left\{y_{n}\right\}$ of points and $\left\{g_{n}\right\}$ of maps such that $x_{n}, y_{n} \in \Lambda\left(g_{n}\right)$ for every $n>0, y_{n} \in W_{\varepsilon}^{\mathrm{ss}}\left(x_{n} ; g_{n}\right)$, $g_{n}$ approaches $f$ and $d\left(x_{n}, y_{n}\right) \geq \delta$ for every $n$. Passing to subsequences, one can suppose that $x_{n} \rightarrow x, y_{n} \rightarrow y$ and $g_{n} \rightarrow f$, thus arriving at a contradiction because $y$ must belong to $W_{\varepsilon}^{\text {ss }}(x ; f) \cap \Lambda$ and $x \neq y$.

Adapting the results of Przytycki ([P1, Section 2]), the local stable manifolds are defined and unique in $\Lambda(g)$. Let $x, y \in \Lambda(g)$ be such that $x \in W_{\varepsilon}^{\mathrm{s}}(y)$. 
Then $d\left(g^{n}(x), g^{n}(y)\right)$ decreases exponentially; moreover, it decreases more in the strong stable direction than in the central one. This will be precisely stated below.

Let there be given a continuous family $\left\{\mathcal{C}_{x}: x \in \Lambda(g)\right\}$ of closed cones, each containing $E_{x}^{\mathrm{ss}}$ in its interior and intersecting $E_{x}^{\mathrm{c}}$ only at the origin.

Lemma 3. There exists some $\delta_{1}>0$ such that if $x, y \in \Lambda(g)$ and $d(x, y)<\delta_{1}$, then $y \notin \widetilde{\mathcal{C}}_{x}^{0}\left(\varepsilon_{0}\right)$.

Proof. On the contrary, assume that there exist sequences $\left\{x_{n}\right\}$ and $\left\{y_{n}\right\}$ in $\Lambda(g)$ such that $y_{n} \in \widetilde{\mathcal{C}}_{x_{n}}^{0}\left(\varepsilon_{0}\right)$ for every $n>0$ and $d\left(x_{n}, y_{n}\right) \rightarrow 0$. By the local product structure it can also be assumed that $y_{n} \in W_{\varepsilon}^{\mathrm{s}}\left(x_{n}\right)$. Moreover, taking suitable preimages as in Remark 1, it follows that there exist sequences of positive integers $m_{n}$ annd points $x_{n}^{m_{n}}$ and $y_{n}^{m_{n}}$ such that

$$
g^{m_{n}}\left(x_{n}^{m_{n}}\right)=x_{n}, \quad g^{m_{n}}\left(y_{n}^{m_{n}}\right)=y_{n}, \quad \delta \leq d\left(x_{n}^{m_{n}}, y_{n}^{m_{n}}\right) \leq \varepsilon,
$$

where $m_{n}$ is minimal with this property.

Observe that $y_{n}^{m_{n}} \in W_{\varepsilon}^{\mathrm{s}}\left(x_{n}^{m_{n}}\right)$. Moreover, the assumption $y_{n} \in \widetilde{\mathcal{C}}_{x_{n}}^{0}\left(\varepsilon_{0}\right)$ implies that

$$
y_{n}^{m_{n}} \in \widetilde{\mathcal{C}}_{x_{n}^{m_{n}}}^{m_{n}}\left(\varepsilon_{0}\right) \subset \widetilde{\mathcal{C}}_{x_{n}^{m_{n}}}^{0}\left(\varepsilon_{0}\right) .
$$

Let $x$ (resp. $y$ ) be a limit point of the sequence $x_{n}^{m_{n}}$ (resp. $y_{n}^{m_{n}}$ ).

If $y \notin \widetilde{\mathcal{C}}_{x}^{k}\left(\varepsilon_{0}\right)$ for some $k>0$, then $y_{n}^{m_{n}} \notin \widetilde{\mathcal{C}}_{x_{n}^{m_{n}}}^{k}\left(\varepsilon_{0}\right)$, which contradicts (9) if $m_{n}>k$. Consequently, $y \in \widetilde{\mathcal{C}}_{x}^{k}\left(\varepsilon_{0}\right)$ for every $k>0$, which implies, by (8), that $y \in W_{\varepsilon}^{\mathrm{ss}}(x) \cap B\left(x ; \varepsilon_{0}\right)$, and this contradicts condition $\mathrm{C}$.

Finally, we obtain the local injectivity of $g$ restricted to $\Lambda(g)$ at $x$ :

Lemma 4. Let $g$ be a $C^{1}$ perturbation of $f$ as above. If $x \in \Lambda(g)$, then there exists a neighborhood $U$ of $x$ such that the restriction of $g$ to $\Lambda(g) \cap U$ is injective.

Proof. By taking local charts, assume that $U$ is a subset of $\mathbb{R}^{n}$ and $x=0$. Then there exists a cone $\mathcal{C}$ such that $\mathcal{C}-\{y\} \subset \widetilde{\mathcal{C}}_{y}$ for every $y \in U \cap \Lambda(g)$. By Lemma $3, x-y \notin \mathcal{C}$ if $x, y \in \Lambda(g)$ and $d(x, y)<\delta_{1}$. Hence if $U$ is small, then Lemma 1(a) implies that $g(x) \neq g(y)$.

Proof of Proposition 2. Since $g$ restricted to $\Lambda(g)$ is locally injective, and since $g$ is a $C^{1}$ perturbation (indeed $C^{0}$ is sufficient) of an injective map in the compact set $\Lambda$, it follows that $g$ is injective in $\Lambda(g)$. This proves Proposition 2 and Theorem 1.

4. Dimension two. This section is devoted to proving the following:

THEOREM 4. If $M$ is two-dimensional, and $f \in \operatorname{End}^{r}(M)(r \geq 1)$ is an Axiom A map with no cycles, then $f$ is $C^{1} \Omega$-stable if and only if any infinite basic piece of $\Omega$ is critical point free. 
Observe that condition $\mathrm{C}$ is trivially necessary for $C^{1} \Omega$-stability in dimension two. If a $C^{1} \Omega$-stable map $f$ has critical nonwandering points, then these points are isolated in $\Omega(f)$ and hence all of them are periodic points.

Proof of Theorem 4. The sufficiency is a consequence of Theorem 1. Beginning the proof of the other direction, assume that $\Lambda$ is a hyperbolic basic set with infinitely many points for an Axiom A map $f$ and there exists a critical point $z_{0} \in \Lambda$. Since $z_{0}$ is a critical point, $\Lambda$ cannot be quasi-expanding, so the restriction of $f$ to $\Lambda$ is injective. On the other hand, as $\Lambda$ is infinite, the dimensions of $E^{\mathrm{s}}$ and $E^{\mathrm{u}}$ are both 1 . The proof of the theorem will be completed if a small perturbation $g$ of $f$ can be found with the property that the restriction of $g$ to $\Lambda(g)$ is not injective: this implies that $f$ is not $C^{1} \Omega$-stable. So fix $\mathcal{U}$, a $C^{1}$ neighborhood of $f$.

We claim that there exists $g \in \mathcal{U}$ having a critical periodic point. This is a very particular case of Franks' lemma (see $[\mathrm{F}]$ ). Let $\varepsilon>0$ be such that every $g$ that is $\varepsilon-C^{1}$ close to $f$ belongs to $\mathcal{U}$. The periodic points of $f$ are dense in $\Lambda$, so there exists a periodic point $p$ of $f$, close enough to $z_{0}$, such that $\left\|D f_{p}(e)\right\|<\varepsilon$ for some unit vector $e$. Without changing the orbit of $p$ one can produce an $\varepsilon-C^{1}$ perturbation $g$ of $f$ such that $D g_{p}(e)=0$.

Indeed, let $V$ be a neighborhood of $p$ disjoint from the future orbit of $p$. Choose coordinates $z=(x, y)$ in $V$ such that $p=0, V=B(0 ; \delta), e=\partial / \partial x$ and define $g(z)=f(z)-\varrho(x) D f_{0}(e)$, where $\varrho: \mathbb{R} \rightarrow \mathbb{R}$ is a $C^{1}$ function satisfying $\varrho^{\prime}(0)=1, \varrho(0)=0,\left|\varrho^{\prime}(x)\right| \leq 1$ for every $(x, y) \in V$, and $\varrho(x)=0$ for every $|x| \geq \delta$. Then $g(0)=f(0), g(x)=f(x)$ outside $V, D g_{0}(e)=0$, and $g$ is $\varepsilon-C^{1}$ close to $f$ in $V$. This implies the claim.

Therefore it is no loss of generality to begin with an $f$ having a critical periodic point $p$ in $\Lambda$. The stable manifold $W^{\mathrm{s}}(p)$ is dense in $\Lambda$. Since $\Lambda$ is an infinite set and $f$ is injective in $\Lambda$, it follows that $W^{\mathrm{s}}(p)$ must intersect $\Lambda$ in some other points (other than $p$ ). Therefore there exist $\varepsilon>0$ such that $W_{\varepsilon}^{\mathrm{s}}(p)$ contains points homoclinic to $p$. This cannot be destroyed by small perturbations. The conditions used in Example 3 of Section 2 are present in this general case:

1. The restriction of $f$ to the local stable manifold of $p$ is non-injective (or can be a made non-injective by a small perturbation).

2. The intersection of this local stable manifold with $\Lambda$ contains more than the point $p$, and this is persistent.

To apply the reasoning of Example 3, first consider an iterate of $f$ such that $p$ is its fixed point; this does not represent any extra assumption since the following perturbations have arbitrarily small support. Take local coordinates such that $p=0$, assume that the local stable manifold of 0 is the axis $y=0$ and that the horizontal foliation is $f$-invariant; it follows that $f(x, y)=\left(f_{1}(x), f_{2}(x, y)\right)$. Only $f_{1}$ will be perturbed. Clearly $f_{1}^{\prime}(0)=0$; 
perturb first $f_{1}$ so that $f_{1}^{\prime}$ is injective in a small neighborhood of 0 . This implies that $f_{1}$ is not injective near 0 . Assume for example that $f_{1}^{\prime}$ is increasing there. Now let $h_{a}(x)=f_{1}(x)-a x, a>0$. Note that $h_{a}(0)=f_{1}(0)$ for every $a$ and let $c_{a}$ be the critical point of $h_{a}$. It is easy to see that $c_{a}$ is well defined, positive and depends continuously on $a$. As $h_{a}^{\prime}$ is injective close to 0 , there exists, for every $a$, a point $x_{a}$ such that $h_{a}\left(x_{a}\right)=h_{a}(0)$. As in Example 3, there exists some value of $a$ such that a homoclinic point of the map $g_{a}(x, y)=\left(h_{a}(x), f_{2}(x, y)\right)$ has the same image as 0 . This shows that $g_{a}$ cannot be conjugate to $f$, and finishes the proof of the theorem.

\section{References}

[AMS] N. Aoki, K. Moriyasu and N. Sumi, $C^{1}$-maps having hyperbolic periodic points, Fund. Math. 169 (2001), 1-49.

[BKR] R. Bamón, J. Kiwi and J. Rivera, Wild Lorenz like attractors, preprint available at www.arxiv.org/math.DS/0508045.

[F] J. Franks, Necessary conditions for stability of diffeomorphisms, Trans. Amer. Math. Soc. 158 (1971), 301-308.

[I] H. Ikeda, $\Omega$-inverse limit stability theorem, ibid. 349 (1996), 2183-2200.

[M] R. Mañé, Axiom A for endomorphisms, in: Lecture Notes in Math. 597, Springer, New York, 1977, 379-388.

[MP] R. Mañé and C. Pugh, Stability of endomorphisms, in: Dynamical SystemsWarwick 1974, Lecture Notes in Math. 468, Springer, New York, 1975, 175-184.

[P1] F. Przytycki, Anosov endomorphisms, Studia Math. 58 (1976), 249-285.

[P2] -, On $\Omega$-stability and structural stability of endomorphisms satisfying Axiom A, ibid. 60 (1977), 61-77.

[T] M. Tsujii, Fat solenoidal attractors, Nonlinearity 14 (2001), 1011-1027.

Instituto de Matemática

Universidade Federal Fluminense

Rua Mario Santos Braga S.N.

CEP 24020-140 Niterói (RJ), Brasil

E-mail: jdelgado@mat.uff.br

Centro de Matematica

Facultad de Ciencias

Universidad de La República

Iguá 4225, C.P. 11400

Montevideo, Uruguay

E-mail: leva@cmat.edu.uy
Departamento de Matemáticas, apdo. 400 Decanato de Ciencias y Tecnología Universidad Centroccidental Lisandro Alvarado Barquisimeto, Venezuela E-mail: nromero@uicm.ucla.edu.ve

Departament de Matemàtica Aplicada 2 Escola Tècnica Superior D'Enginyeria Industrial Universidad Politècnica de Catalunya Colom 11, 08222 Terrasa, Barcelona, Spain E-mail: fvilamajo@upc.es

Received 24 November 2005; in revised form 15 September 2006 